\title{
Low Cost and High Efficiency Hybrid Architecture Massive MIMO Systems Based on DFT Processing
}

\author{
Weiqiang Tan $\left(\mathbb{D},{ }^{1}\right.$ Guixian $X{ }^{2},{ }^{2}$ Elisabeth De Carvalho, ${ }^{2}$ Mu Zhou $\left(\mathbb{D},{ }^{3}\right.$ \\ Lisheng Fan $\mathbb{B}^{1},{ }^{1}$ and Chunguo $\mathrm{Li}\left(\mathbb{1}^{4}\right.$ \\ ${ }^{1}$ School of Computer Science and Educational Software, Guangzhou University, Guangzhou 510006, China \\ ${ }^{2}$ Department of Electronic Systems, Aalborg University, 9400 Aalborg, Denmark \\ ${ }^{3}$ Chongqing Key Lab of Mobile Communications Technology, Chongqing University of Posts and Telecommunications, \\ Chongqing 400065, China \\ ${ }^{4}$ National Mobile Communications Research Laboratory, Southeast University, Nanjing 210096, China
}

Correspondence should be addressed to Lisheng Fan; lsfan@gzhu.edu.cn

Received 24 April 2018; Revised 10 July 2018; Accepted 2 August 2018; Published 14 August 2018

Academic Editor: Zheng Chu

Copyright (C) 2018 Weiqiang Tan et al. This is an open access article distributed under the Creative Commons Attribution License, which permits unrestricted use, distribution, and reproduction in any medium, provided the original work is properly cited.

Low cost and high efficiency, defined as energy efficiency (EE) and spectral efficiency (SE), have raised more and more attention in the fifth generation $(5 \mathrm{G})$ communication systems due to steadily rising hardware cost, energy consumption, and mobile traffic. This paper studies the hybrid architecture of multiuser massive MIMO systems, where the digital domain utilizes the zero-forcing (ZF) precoding scheme and the analog domain uses discrete Fourier transform (DFT) processing that significantly reduces hardware cost and energy consumption. We derive analytical expressions on the total achievable SE and EE, as well as offering insight into some engineering parameters in the system performance. Our aim is to achieve low cost and high efficiency massive MIMO system, with constraints on the overall transmit power, the number of users, and the number of radio frequency (RF) chains. Results exhibit that the total achievable SE of the hybrid architectures with DFT precessing is inferior to the full digital architectures and hybrid architectures with the ideal phase shifters, but the performance attenuation can be compensated by providing the more input SNR and higher number of RF chains. Moreover, we find that the total achievable EE of hybrid architectures with DFT precessing outperforms other massive MIMO architectures that include a full digital implementation, ideal phase shifters, and a switched network.

\section{Introduction}

As environmental issue has attracted more and more concern from public, green radio communication has emerged as the most important concept in the fifth generation (5G) communication systems [1-4]. Along with improved traffic rate, transmission reliability, and quality of service, the upcoming $5 \mathrm{G}$ communication systems aim at improving the energy efficiency without compromising on the user experience [5-8]. Massive multiple-input multiple-output (MIMO), viewed as one of the important techniques for $5 \mathrm{G}$ wireless communication, has attracted a great deal of research interest in current years. For a conventional massive multiple-input multiple-output (MIMO) system with fully digital architecture, every antenna element requires to deploy a radio frequency $(\mathrm{RF})$ chain that is equipped with a highresolution analog-to-digital converter (ADC). The energy consumption of the ADCs grows exponentially with the number of ADC quantization bits and also increases with carrier frequency bandwidth and signal sampling rate. However, massive MIMO systems employing large-scale number antennas and high-resolution ADCs lead to unaffordable energy consumption and hardware cost [9-12].

Hybrid architectures massive MIMO systems, which consist of analog processing and digital precoding, are one such alternative with promising techniques in $5 \mathrm{G}$ communication systems [13-15]. Such architectures not only achieve high total achievable SE, but also keep low cost and energy consumption. There has been a lot of work devoted to developing analog beamforming design. For example, the 
authors in $[16,17]$ proposed a novel algorithm for pointto-point MIMO systems, which aimed to reduce the computational complexity of system and maximize the total achievable spectral efficiency (SE) on different subcarriers. To handle the severe energy consumption and hardware cost, the authors in [18] proposed user scheduling algorithms and resource allocation schemes, which aimed to maximize the total achievable energy efficiency (EE) on hybrid massive system. However, all the aforementioned studies are based on the ideal phase shifters at the analog domain, which has a potential to enhance the complexity of the system, especially for a huge quantity of data. In order to simplify the complexity of system, the work of [19] proposed hybrid architecture with a switches network, where the ideal phase shifters are replaced by a switches network. Results reported that the switches network is a simpler solution but suffers from the significantly performance loss of the system.

Fortunately, the work of [20] found that using discrete Fourier transform (DFT) processing at analog domain has similar nature with electromagnetic lens and outperforms the fully connected phase shifters, which has low power consumption and is easy to implement. In light of these benefits, the DFT processing at the analog domain has gained great attention [21]. Results in [22] demonstrated that the DFT processing is carried out by the Butler circuit, which has low cost and high energy efficiency. Most importantly, the authors in [23] reported that the DFT processing is treated as the most promising technique used for signal transfer, which has been recommended by various industrial ports. Meanwhile, the authors in [24] investigated hybrid massive MIMO system based on DFT processing and further obtained the bounds on the achievable SE of such system, where the zero-forcing (ZF) precoding is adopted by the baseband processing. These studies confirm that the hybrid massive MIMO system based on DFT processing provides advantages for massive MIMO systems. However, the aforementioned work of $[23,24] \mathrm{did}$ not derive an exact expression on the achievable SE and did not include analytical results on the achievable EE for hybrid massive MIMO systems.

In order to compensate for this gap in the study of achievable SE and EE, this paper presents a novel hybrid analog/digital architecture for massive MIMO systems, where analog processing is implemented using DFT processing. Moreover, we further investigate the EE and SE of the hybrid architecture massive MIMO system, which aims to effectively reduce the power consumption and hardware cost without compromising on the achievable SE. Our main contributions are as follows:

(i) We present a novel hybrid architecture massive system based on the DFT processing and derive exact and approximated expressions on the total achievable $\mathrm{SE}$ of system. According to the derived analytical result, we further offer insight into the impact of several engineering parameters in the system performance, which constraints on the overall transmit power, the number of users, and the number of RF chains. (ii) By establishing the realistic power consumption model and employing the derived approximate expression, we evaluate the total EE for hybrid architectures with DFT processing. Closed-form expressions for the optimal the number of RF chains and the SNR by maximizing the achievable EE are derived.

(iii) Results exhibit that the total achievable SE of the hybrid architectures with DFT precessing is inferior to the full digital architectures and hybrid architectures with the ideal phase shifters, but the performance loss can be compensated by offering more RF chains and more SNR. We also find that hybrid architectures with DFT precessing exhibit much better total achievable EE than the other hybrid architectures massive MIMO systems.

The reminder of the paper is listed as follows. Section 2 presents the signal model and hybrid architectures massive MIMO system with DFT precessing. In Section 3, we analyze the system's performance that includes the achievable SE and EE. Based on the derived theoretical results, we attain the several engineering insights by considering the special cases. Section 4 provides the numerical results to confirm the obtained analytical results. Section 5 outlines the whole paper.

Notations. Matrix and vector are used by upper and lower case boldface, $\operatorname{det}(\cdot), \operatorname{diag}(\cdot)$, and $(\cdot)$ ! stand for a matrix determinant, diagonal matrix, and factorial operation, respectively, $[\mathbf{G}]_{k, k}$ denotes the $k$-th diagonal elements of $\mathbf{G},\lfloor\cdot\rfloor$ accounts for rounding to the nearest integer, and $\mathbf{I}_{K}$ and $\mathbb{C}^{n \times n}$ account for an identity matrix of $K \times K$ dimension and an complex Gaussian matrix of $n \times n$ dimension, respectively.

\section{System Model}

We firstly present the signal model associated with a hybrid analog/digital architecture in massive MIMO system, where the analog domain utilizes the low cost DFT processing, and then we describe channel model that consists of the small and large fading.

2.1. Signal Model. As depicted in Figure 1, we present a hybrid architecture of a multiuser massive MIMO system, where the BS comprises $N_{t}$ transmit antennas simultaneously serving $M$ users. Each user has a single-antenna element and the $N_{t}$ transmit antennas are connected to $N_{s}$ RF chains via a DFT processing with parameters restricted as $M \ll N_{t}$ and $M \leq N_{s} \leq N_{t}$. Assume that $\mathbf{x}$ denotes a transmitted signal vector of $M \times 1$ dimension for all users with $\mathbb{E}\left[\mathbf{x x ^ { H }}\right]=\mathbf{I}_{M}$. When the input signal is the Gaussian symbol [25-27], the received signal after analog DFT processing technique and digital linear precoding scheme can be given by

$$
\mathbf{y}=\sqrt{P} \mathbf{G}^{H} \mathbf{F W} \mathbf{x}+\mathbf{n},
$$

in which $P$ stands for the input SNR of the system, W represents the digital baseband precoder matrix, $\mathbf{F}$ denotes the analog processing matrix, $\mathbf{n}$ denotes the additive white Gaussian noise (AWGN), and $\mathbf{G}$ accounts for the channel 


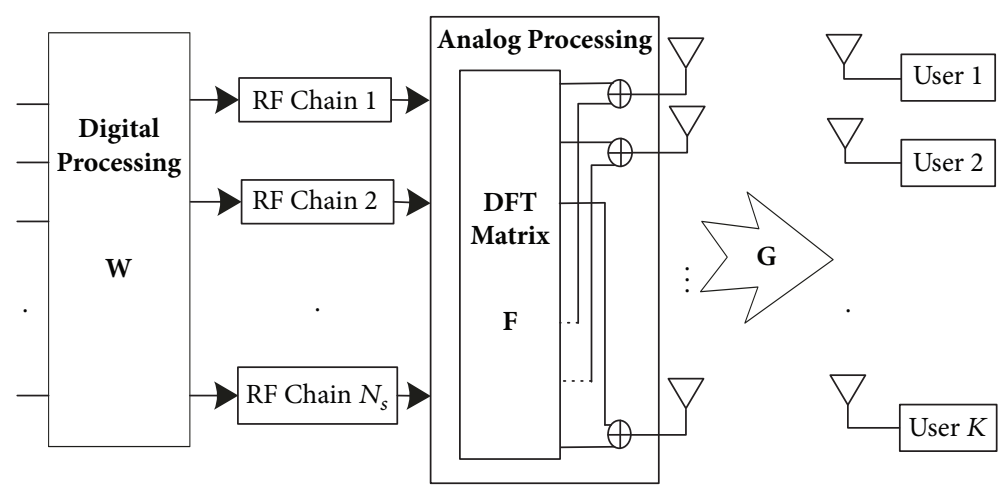

FIGURE 1: Illustration of hybrid architecture massive MIMO system, in which the analog domain adopts DFT processing.

matrix that includes the large-fading coefficient and fastfading coefficient, which can be given by

$$
\mathbf{G}=\mathbf{H D}^{1 / 2}
$$

where $\mathbf{H}$ accounts for the small-scale fading matrix, whose entries follow a complex Gaussian distribution, and D denotes large-scale fading matrix that diagonal entries are given by $[\mathbf{D}]_{k, k}=\beta_{k}$ and nondiagonal entries equal zero. In practical, large-scale fading coefficient $\beta_{k}$ contains the shadowing fading and path loss of the user $k$, which is modeled as $\beta_{k}=z_{k} r_{k}^{-\gamma}$, where $z_{k}$ and $\gamma$ account for a lognormal random variable of the user $k$ and the decay exponent of path loss, respectively, and $r_{k}$ accounts for the distance between the BS and the user $k$.

2.2. DFT Processing Implemented. In the previous works about hybrid massive MIMO systems [13, 28], phase shifters or a network of switches requires the ideal CSI to be available at the BS. To overcome this drawback brought by phase shifters, this paper investigates a hybrid massive MIMO system as showed in Figure 1, in which the analog domain is implemented by using DFT processing. For hybrid architecture with DFT processing, all transmitted signals via a partial DFT matrix preprocessing before digital baseband precoding, in which the partial DFT matrix is derived by uniformly randomly selecting the rows of a DFT matrix. Therefore, the partial matrix $\mathbf{F}$ is constructed by

$$
\mathbf{F}=\frac{1}{\sqrt{N_{t}}}\left[\begin{array}{ccccc}
1 & 1 & 1 & \cdots & 1 \\
1 & w & w^{2} & \cdots & w^{\left(N_{s}-1\right)} \\
1 & w^{2} & w^{4} & \cdots & w^{2\left(N_{s}-1\right)} \\
\vdots & \vdots & \vdots & \ddots & \vdots \\
1 & w^{\left(N_{t}-1\right)} & w^{2\left(N_{t}-1\right)} & \cdots & w^{\left(N_{t}-1\right)\left(N_{s}-1\right)}
\end{array}\right],
$$

where $w=e^{-2 \pi i / N_{t}}$.

Remark 1. From energy efficiency and economic perspective, the DFT processing is appealing for analog domain in massive MIMO systems since it has low energy consumption, less attenuation, and high performance, while the DFT processing is implemented by adopting a field programmable gate array (FPGA) [29] or an integrated Butler circuit [30], which is easy to implement and efficiently reduces hardware complexity. In addition, from the actual practical implementation, the DFT processing able to embrace all propagation signal from $K$ users and the DFT processing is implemented offline, which makes sure all RF chains always be activated.

\section{Performance Analysis of System}

In this section, our main task aims to derive the exact expression on the achievable SE. By using the obtained theoretical result, we offer the insight of engineering parameters on the achievable SE. And then the total achievable EE is investigated by considering the energy consumption of such a system. Finally a brief discussion is provided on how to achieve the EE maximization.

3.1. Hybrid Massive MIMO System. A hybrid architecture massive MIMO system with DFT processing is displayed in Figure 1, which is split into DFT processing and digital precoding processing successively. Before DFT processing at the analog domain and digital precoding at the baseband domain, an equivalent channel matrix $\mathbf{G}_{e q}$ is equivalent to a composite matrix composed of the channel matrix and partial DFT matrix, i.e., $\mathbf{G}_{e q}=\mathbf{G}^{H} \mathbf{F}$. Thus, the digital precoding matrices are based on $\mathbf{G}_{e q}$. In addition, we make an assumption that the accurate and instantaneous CSI is available. In practical setups, the CSI is efficiently derived by channel estimation [31] and shared with the users using limited feedback techniques such as given in [32]. The ZF precoding scheme is able to completely eliminate interference among users, which achieves the performance of the nonlinear precoding schemes, for example, dirty paper coding (DPC) [33]. Thus, we would like to employ zero-forcing (ZF) precoding at the digital domain; $\mathbf{W}$ can be written as

$$
\mathbf{W}=\mathbf{G}_{e q}^{H}\left(\mathbf{G}_{e q} \mathbf{G}_{e q}^{H}\right)^{-1} \boldsymbol{\Xi},
$$

where $\boldsymbol{\Xi}$ accounts for the normalization matrix that satisfies the constraint condition, i.e., $[\boldsymbol{\Xi}]_{k, k}=1 / \sqrt{\left[\mathbf{W}^{H} \mathbf{W}\right]_{k, k}}$, which makes sure the precoding power constraint is satisfied. When the input symbol is a Gaussian random variable, the 
achievable SE of the user $k$ with a ZF precoding scheme is expressed as

$$
R_{k}=\mathbb{E}\left\{\log _{2}\left(1+\frac{1}{\left[\left(\mathbf{G}_{e q} \mathbf{G}_{e q}^{H}\right)^{-1}\right]_{k, k}}\right)\right\} .
$$

Considering all the users, the total achievable ergodic SE of system is identical to a sum of the achievable ergodic SE per user, which is calculated as

$$
R_{\text {sum }}=\sum_{k=1}^{M} R_{k} .
$$

According to (5), we see that the expectation is carried out via ergodic of the equivalent channel matrix. In order to derive the exact expression of the achievable SE, we have to calculate the probability density function (p.d.f) of the SINR term. In the following section, we shall present the detailed process about the achievable SE.

\subsection{Spectral Efficiency Analysis}

Theorem 2. Consider hybrid architecture massive MIMO systems with DFT processing; the exact expression on the achievable ergodic SE of the user $k$ with ZF precoding scheme is calculated as

$$
R_{k}=\log _{2}(e) e^{1 / P \beta_{k}} \sum_{h=1}^{N_{s}-M+1} E_{h}\left(\frac{1}{P \beta_{k}}\right),
$$

where $E_{h}(\cdot)$ denotes the exponential integral function of order $h$. For real nonzero values of $x$ and the integer $h$, the exponential integral $E_{h}(x)$ is defined as

$$
E_{h}(x)=\int_{1}^{\infty} \frac{e^{-x t}}{t^{h}} d t .
$$

Proof. See Appendix A.

From Theorem 2, it is shown that the achievable SE of the user $k$ is related to the number of RF chains, the input SNR, and the number of users. However, it is hard to get an intuition about the impact of the system parameters on the achievable SE, since it invokes the exponential integral function. Therefore, we try to calculate an approximate expression on the achievable SE, which enables us to directly observe the general trends of the SE as a function of the most determinant parameters.

Corollary 3. Considering a hybrid architecture massive MIMO system based on DFT processing, an approximate expression on the achievable $S E$ can be given by

$$
\bar{R}_{k}=\log _{2}\left(1+P \beta_{k}\left(N_{s}-M+1\right)\right) .
$$

Proof. To begin with, we invoke the results in [34] and directly obtain the following approximate expression:

$$
e^{1 / x} \sum_{n=1}^{N-1} E_{n}\left(\frac{1}{x}\right) \approx \log (1+x(N-1)) .
$$

According to the above approximate equation, we have the following result:

$$
\begin{aligned}
& e^{1 / P \beta_{k}} \sum_{h=1}^{N_{s}-M+1} E_{h}\left(\frac{1}{P \beta_{k}}\right) \\
& \approx \log \left(1+P \beta_{k}\left(N_{s}-M+1\right)\right) .
\end{aligned}
$$

Substituting (11) into (8), we can derive the desired result.

With the derived tractable expression in (9) at hand, we obtain clear insights into the SE behavior with respect to different system parameters. From (9), we observe that $\bar{R}_{k}$ is concerned with the large-fading coefficient, the input SNR, the number of RF chains, and the number of users. It can be seen that $\bar{R}_{k}$ is a monotonically increasing function of the number of RF chains and the input SNR. Additionally, assuming a fixed number of BS antennas and the input SNR, $\bar{R}_{k}$ is a monotonically decreasing function of the number of users. However, as the number of users grows, the total achievable SE increases since it is the multiplication of the achievable ergodic per rate by $M$. Besides, the total achievable $\mathrm{SE}$ is a concave function with respect to the number of users $M$, and there exists a unique globally optimal number of users maximizing the total achievable SE according to convex optimization theory. In the following corollary, we keep the other parameters being fixed and concentrate on finding the best number of users to achieve the total achievable SE maximization.

Corollary 4. For hybrid architecture massive MIMO systems based on DFT processing, there exists a unique globally optimal number of users $M^{\text {opt }}$ that achieve the total achievable SE maximization, which is

$$
M^{o p t}=\left\lfloor\frac{\Delta}{P \beta}\left(1-\frac{1}{W_{0}(\Delta \exp (1))}\right\rfloor,\right.
$$

where $\Delta=1+P \beta\left(N_{s}+1\right)$ and $W_{0}(\cdot)$ represents the Lambert function.

Proof. See Appendix B.

Corollary 4 reveals an important conclusion that the achievable SE will not linearly increase with the number of users, but it is a convex function. This implies that the optimal number of users $M^{\text {opt }}$ in (12) achieves the total achievable SE maximization. Additionally, it worth noting that Corollary 4 typically shows us a noninteger value with respect to $\mathrm{M}^{\text {opt }}$; however, the quasi-concavity of the solution in (12) indicates that $M^{\text {opt }}$ is obtained at one of the two closest integers.

3.3. Energy Efficiency Analysis. Green radio communication has emerged as a new prominent indicator in the design of $5 \mathrm{G}$ communication systems $[35,36]$. Thus, the total achievable EE has become a primary concern, which can be viewed as the total achievable SE divided by the total energy consumption, which is established as

$$
\eta_{E}=\frac{B R_{\text {sum }}}{P_{\text {total }}}
$$


where $B$ stands for the available bandwidth, whose unit is $\mathrm{MHz}, P_{\text {total }}$ accounts for the overall energy consumption, and $R_{\text {sum }}$ was defined as the total achievable SE of system, which is provided in (6).

We now present a practical energy consumption study of the system. According to the prior works of [37-39], the main energy consumption comes in several varieties: circuit energy $P_{\mathrm{C}}$, signal processing energy $P_{\mathrm{SP}}$, and signal transmission energy $P_{\mathrm{T}}$. Therefore, the total energy consumption in our system can then be calculated as

$$
P_{\text {total }}=P_{\mathrm{C}}+P_{\mathrm{SP}}+P_{\mathrm{T}}
$$

Next, we shall introduce a detailed explanation of each energy consumption part in the following.

(1) Circuit energy: the circuit loss of system mainly comes from three parts: which includes the LNA module, DFT processor, and one RF chain. Thus, the circuit energy is calculated as

$$
P_{\mathrm{C}}=N_{t} P_{\mathrm{LAN}}+P_{\mathrm{DFT}}+N_{s} P_{\mathrm{RF}}
$$

where $P_{\text {LAN }}$ accounts for the energy consumption from a LNA, $\mathrm{P}_{\mathrm{DFT}}$ denotes the maximum energy consumption from DFT processor at the analog domain, and $P_{\mathrm{RF}}$ is the energy consumption for a single RF chain.

(2) Signal processing energy: the total energy consumption mainly comes from the signal processing, which is given by [39]

$$
P_{\mathrm{SP}}=K P_{\mathrm{cd}}+\left(\frac{2 M^{2} N_{t}+2 M N_{t}}{L T}+\frac{2 M^{3}}{3 L T}\right)+\frac{N_{t} M}{L},
$$

where $P_{\mathrm{cd}}=P_{\text {code }}+P_{\mathrm{dec}}, P_{\text {code }}$ and $P_{\mathrm{dec}}$ stand for the energy consumption caused by the coding and decoding symbols, respectively. The second term can be understood as the energy computation of precoding due to the matrix inversion of the $\mathrm{ZF}$ scheme. The values of $T, L$, and $U$ stand for the coherence time, the computational efficiency, and the number of blocks per sec, respectively. The third term can be understood as the energy computation of information symbols during signal transmission.

(3) Signal transmission energy: the energy consumption mainly comes from the loss of signal transmission in the circuits and transmission, which is function of the input SNR and is equivalent to $P_{\mathrm{T}}=P / \eta$, where $\eta$ accounts for the effective coefficient of the input SNR.

Under the above consideration about the energy consumption, we shall derive a closed-form expression of total achievable EE. By plugging (14) into (13), the total achievable $\mathrm{EE}$ of system is expressed as

$$
\begin{aligned}
& \eta_{E} \\
& =\frac{B \log _{2}\left(1+P \beta_{k}\left(N_{s}-M+1\right)\right)}{N_{t} I_{1}+N_{s} P_{\mathrm{RF}}+M\left(P_{\mathrm{cd}}+2 M^{2} / 3 L T\right)+P / \eta+P_{\mathrm{DFT}}},
\end{aligned}
$$

where $I_{1}=P_{\mathrm{LAN}}+M / L+2 M^{2} / L T$. Based on the above optimization problem, it is shown that the total achievable EE in (17) is related to other fixed energy consumption, the number of BS antennas, the number of RF chains, the input SNR, and the number of users. Maintaining the number of BS antennas, the number of users, the input SNR, and the number of RF chains constant, we will focus on finding the best input SNR that achieves the total achievable EE maximization.

Corollary 5. Keeping the number of BS antennas, the number of $R F$ chains, the number of users, and the other parameters $\left(B, I_{1}, L, T, \beta_{k}, \eta, P_{R F}, P_{c d}, P_{D F T}\right)$ constant, the optimal SNR is calculated as (18), which can be found below.

$$
P^{o p t}=\frac{\exp \left\{W_{0}\left(\eta \beta_{k}\left(N_{s}-M+1\right)-1 /\left(N_{t} I_{1}+N_{s} P_{R F}+M\left(P_{c d}+2 M^{2} / 3 L T\right)+P_{D F T}\right)+1\right)\right\}-1}{\beta_{k}\left(N_{s}-M+1\right)}
$$

Proof. To complete the proof, we consider the optimization function $g(x)$ as follows:

$$
\max g(x)=\frac{f \log _{2}(a+b x)}{c+d x}
$$

with constants $a, c \geq 0$ and $b, d, f>0$. With the aid of the conclusion provided in [40], we infer that the objective function is strictly quasi-concave and there exists an optimal $x$ that maximizes $g(x)$; the optimal $x$ can be given by

$$
x^{\mathrm{opt}}=\frac{e^{W_{0}((b c-a d) / d e)+1}-a}{b},
$$

where $e$ is the natural number. By capitalizing on the above result and keep the system parameters constant except the $\mathrm{SNR}$, we arrive at the desired result.

Corollary 5 provides an explicit guideline on how much SNR should be offered at the BS to maximize the EE. But, it is worth noting that the optimal SNR involves the Lambert function $W(x)$, which is a strictly increasing formula in accordance with the random variance $x[41,42]$. In the following, we shall present the numerical result to verify the derived analytical result.

Corollary 6. Keeping the number of BS antennas, the $S N R$, the number of users, and the other parameters 
$\left(B, I_{1}, N_{t}, L, T, \beta_{k}, \eta, P_{R F}, P_{c d}, P_{D F T}\right)$ as constant, the number of $R F$ chains is calculated as

$$
\begin{aligned}
& N_{s}^{o p t}=\left\lfloor\left(\frac{1}{P \beta_{k}}\right)\right. \\
& \cdot \exp \left(W_{0}\left(\frac{P \beta_{k}\left(2 M^{3} / 3 L T+P / \eta+N_{t} I_{1}+I_{2}\right)}{P_{R F} e}\right)\right. \\
& +1)+(M-1)\rfloor
\end{aligned}
$$

where $I_{2}=M P_{c d}+P_{D F T}-P_{R F}(1-M)$.

Proof. Using the similar method, the desired result is directly obtained by directly utilizing the results provided in (20) and setting the other parameters being constant except the number of RF chains.

\section{Numerical Results}

In this section, we provide the Monte-Carlo simulations to confirm the theoretical results on the achievable SE, which have been derived in Section 3, and then we compare the achievable EE for full digital architectures and hybrid architectures by considering realistic power consumption. In all simulations, the cell radius is 1000 meters, the guard zone ring region is set to 100 meters, the number of users is set to eight, the bandwidth of carrier frequency is $B$ $=10 \mathrm{MHz}$, the computational efficiency is $L=12.8$, the coherence time is set to $\mathrm{T}=32 \mathrm{~ms}$, the decay exponent of large-scale fading is given by $\gamma=2.1$, and the standard deviation of large-scale fading is $\sigma_{\text {shad }}=4.9 \mathrm{~dB}$. The largefading coefficients $\beta_{k}(k=1, \ldots, 8)$ are randomly created as follows: $\{3.14,0.09,0.154,5.38,3.29,0.16,11.93,1.82\} \times 10^{-3}$. Unless specified otherwise, the above simulation parameters are used. All the numerical results are averaged over 10,000 independent channel realizations.

Figure 2 depicts the total achievable SE versus the input SNR, where all curves include the exact expression of Theorem 2, the approximate expression of Corollary 3, and the simulated results. We can see that the curves for the simulated results, the approximate expression, and exact expression coincide perfectly in the entire SNR regime. This observation confirms the derived analytical expressions in Theorem 2 and Corollary 3. Meanwhile, Figure 2 also provides the total achievable SE for the full digital architectures [43], as well as the hybrid architectures with the ideal phase shifters and switch network [19]. It is shown that the total achievable SE of full digital architectures always outperforms the one of hybrid architectures, regardless of ideal phase shifters, switch network, and DFT precessing. However, the total achievable SE of the hybrid architectures and DFT precessing is superior to that of the hybrid architectures with the ideal phase shifters, but it is inferior to that of the hybrid architectures with the ideal phase shifters. As expected, there is a significant performance gap that is caused by inaccurate CSI and randomly DFT selection. According to (9), we know

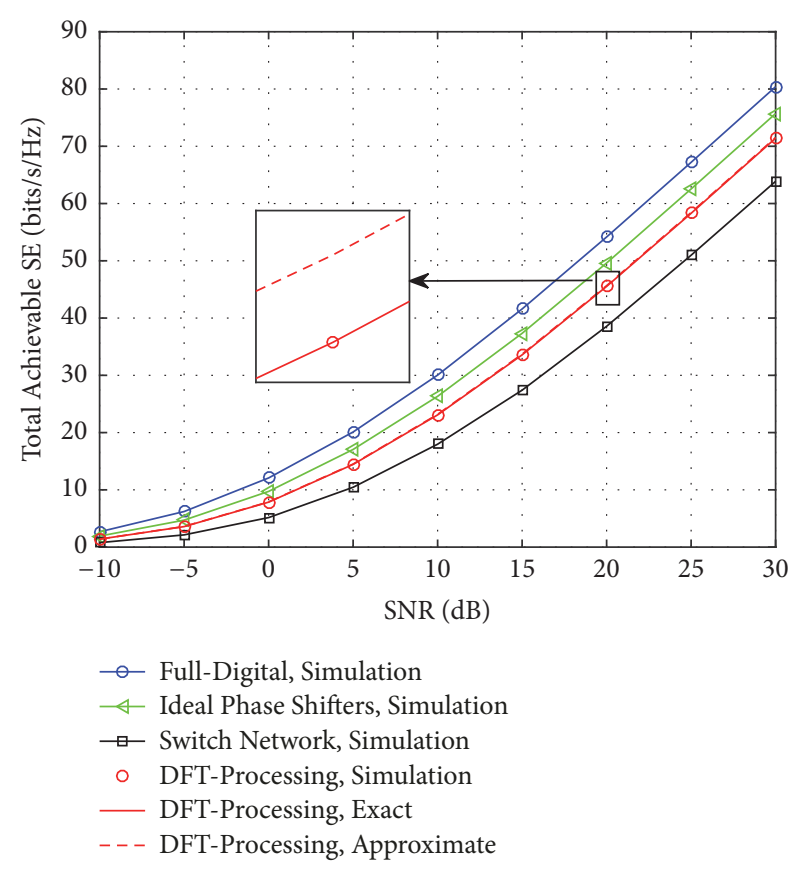

FIgURE 2: Total achievable SE versus the input SNR for different architectures $\left(N_{t}=100, M=8\right.$, and $\left.N_{s}=50\right)$.

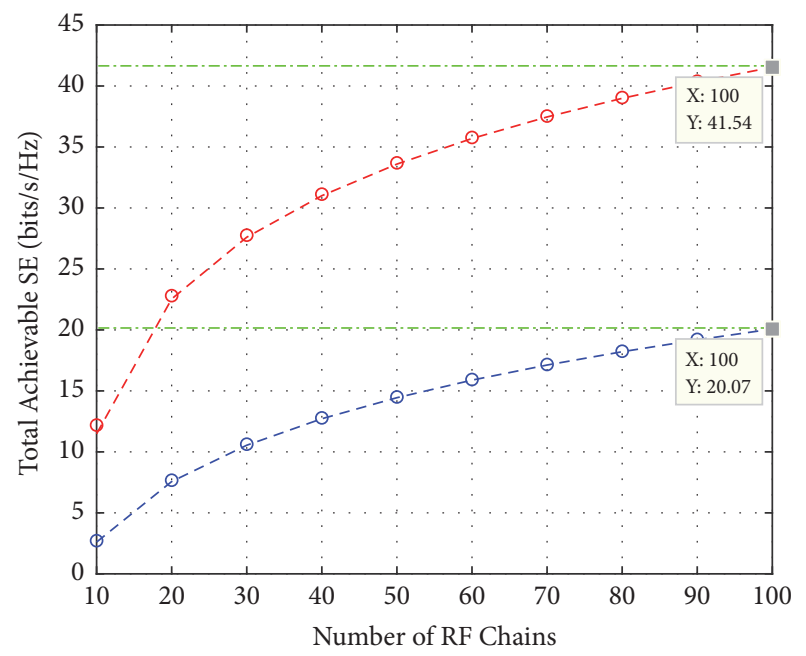

- DFT-Processing, Simulation, $\mathrm{SNR}=15 \mathrm{~dB}$

- - DFT-Processing, Approximate, $\mathrm{SNR}=15 \mathrm{~dB}$

○ DFT-Processing, Simulation, $\mathrm{SNR}=5 \mathrm{~dB}$

- - - DFT-Processing, Approximate, $\mathrm{SNR}=5 \mathrm{~dB}$

FIgURE 3: Total achievable $S E$ versus the number of RF chains $\left(N_{t}=\right.$ $100, M=8$, and SNR $=5 \mathrm{~dB}, 15 \mathrm{~dB}$ ).

that the performance gap can be compensated for offering the more input SNR and the more number of RF chains.

In Figure 3, we depict the total achievable SE versus the number of RF chains. As expected, the total achievable SE increases with the number of RF chains, which keeps pace with theoretical results in Corollary 3. For comparison, the total achievable SE for the full digital architectures is 


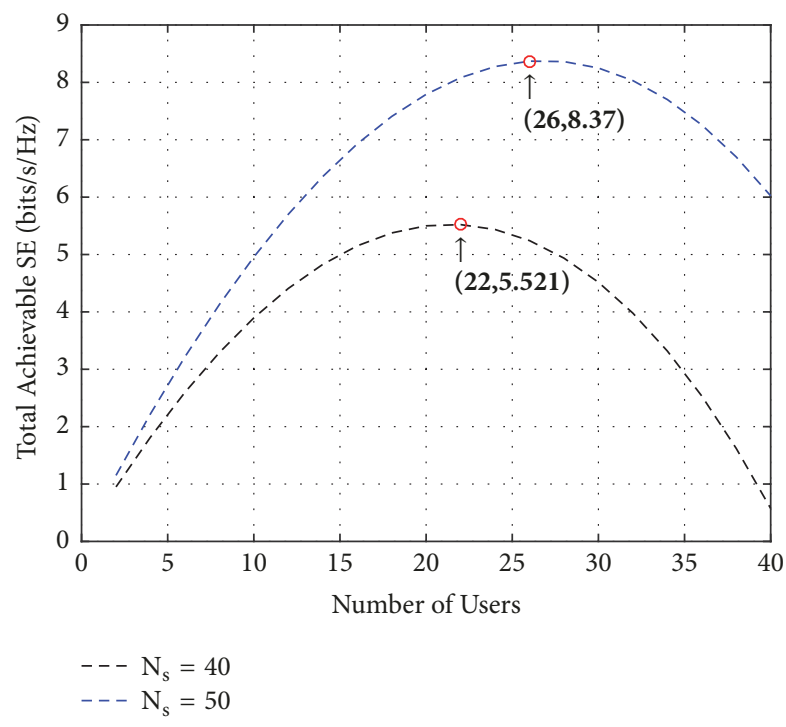

Figure 4: Total achievable SE with DFT processing versus the number of users $\left(N_{t}=128, \mathrm{SNR}=10 \mathrm{~dB}\right.$, and $\left.N_{s}=40,50\right)$.

also provided using [43, Table I], respectively. We can see that, with fixed number of users, number of BS antennas, and SNR (15 dB and $5 \mathrm{~dB})$, the total achievable SE for full digital architectures maintains a constant value and is 41.54 and $20.07 \mathrm{bit} / \mathrm{s} / \mathrm{Hz}$, respectively, which keeps constant. This is because each antenna is connected to the RF chain. In addition, only when the number of available RF chains equals the number of BS antennas $\left(N_{s}=N_{t}=100\right)$, the difference between hybrid architectures and full digital architectures tends to zero. Moreover, we observe that the total achievable SE with larger SNR regime always outperforms that one of the smaller SNR regime for the reason that the larger transmitted power boosts the total achievable SE.

Figure 4 depicts the total achievable SE varying with the number of users, in which the curves only showed the hybrid architecture with DFT processing. To guarantee fairness among users, we assume that the large-scale fading of each user is set to be identical, namely, $\beta_{k}=1 \times 10^{-3}, \forall M$. Moreover, given the number of BS antennas and the input SNR $\left(N_{t}=128\right.$ and SNR $\left.=10 \mathrm{~dB}\right)$, we observe that the total achievable SE increases first and then decreases as the number of users increases from 2 to 40 . Meanwhile, we depict the total achievable SE for different numbers of RF chains $\left(N_{s}=40,50\right)$; no matter what the number of RF chains is utilized, a globally optimal number of users always exists that achieves the total achievable SE optimum, which keeps pace with theoretical result in Corollary 4. In addition, keeping the parameters $N_{s}, P$, and $\beta$ constant and plugging these parameters into (B.1) and (12), the best number of users and corresponding total achievable SE of system is given by $(22$, $5.521)$ and $(26,8.370)$, respectively, which further support our theoretical result in Corollary 4.

In Figures 5 and 6, we compare the total achievable EE for a hybrid massive MIMO system based on DFT processing, ideal phase shifters, and switch network, as well as massive MIMO systems with full digital architecture. Before depicting

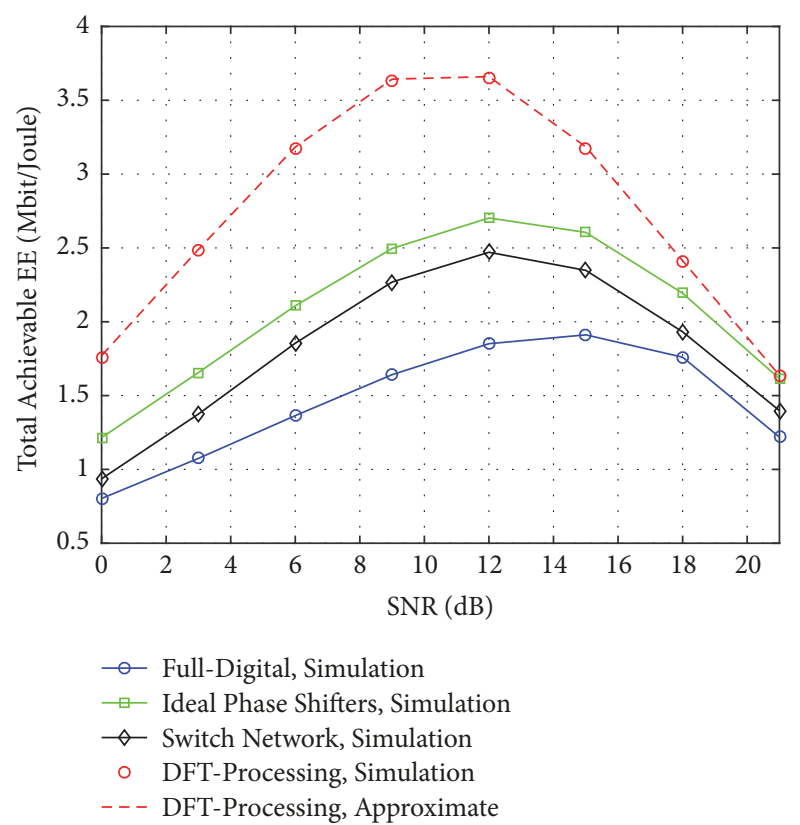

FIgURE 5: Total achievable EE versus SNR for different architectures $\left(N_{t}=128, N_{s}=50\right.$, and $\left.M=8\right)$.

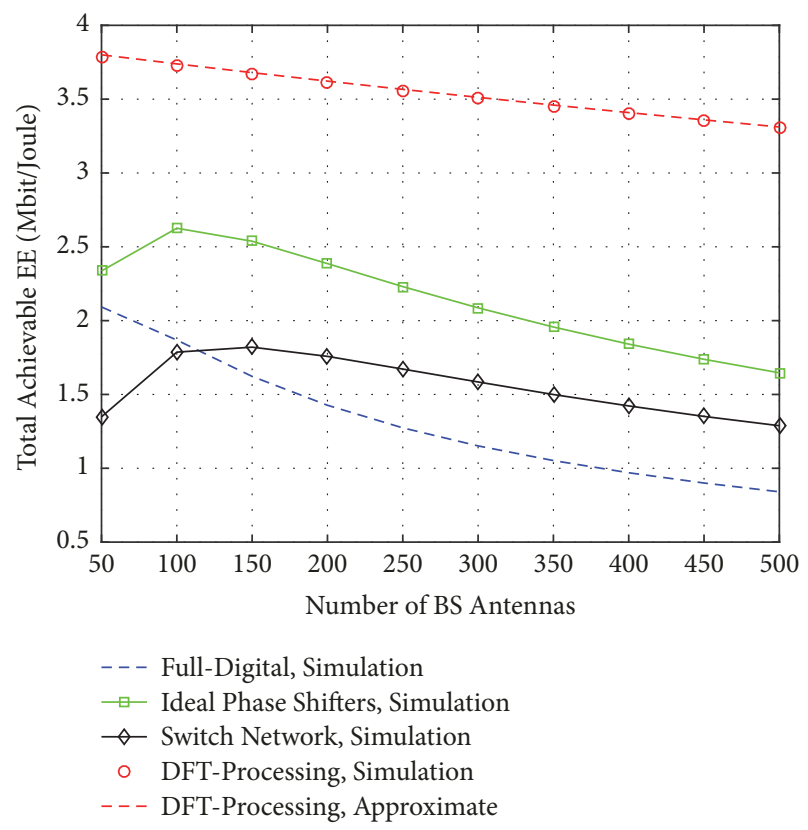

FIGURE 6: Total achievable EE versus the number of BS antennas for architectures $\left(\mathrm{SNR}=10 \mathrm{~dB}, N_{s}=50\right.$, and $\left.M=8\right)$.

the total achievable EE, we shall present the energy consumption for full digital architecture, hybrid architectures with ideal phase shifters, and switch network hybrid architectures because the energy consumption of hybrid architectures with DFT processing has been provided in Section 3. With the help of the energy consumption models that have been provided in $[19,37,44]$, we know that, for full digital architecture, hybrid architectures with ideal phase shifters, and switch network hybrid architectures, the energy consumption of the 
signal processing part and the signal transmission part is the same as the hybrid architectures with DFT processing, and only the energy consumption of the circuit part has changed drastically. For full digital architecture, the energy consumption of the circuit part is provided by [44]

$$
P_{\text {Full }}=N_{t} P_{\mathrm{BS}}+P_{\mathrm{SYS}}+M P_{\mathrm{UE}}
$$

where $P_{\mathrm{BS}}=1$ Watt, $P_{\mathrm{SYS}}=2 \mathrm{Watt}$, and $P_{\mathrm{UE}}=0.3$ Watt. For a hybrid architecture massive MIMO system based on ideal phase shifters and switch network, the energy consumption of circuit part is provided by [19]

$$
P_{* *}=N_{t}(K+1) P_{\mathrm{LAN}}+N_{t} M P_{* *}+M P_{\mathrm{RF}},
$$

where the subscript $* *$ represents two different cases of the ideal phase shifter and switch network. The detailed energy consumption is provided by $P_{\mathrm{LAN}}=20 \times 10^{-3}$ Watt, $P_{\mathrm{PS}}=$ $30 \times 10^{-3}$ Watt, $P_{\mathrm{SW}}=5 \times 10^{-3}$ Watt, $P_{\mathrm{FR}}=40 \times 10^{-3}$ Watt, $P_{\text {cod }}=4$ Watt, and $P_{\text {dec }}=500 \times 10^{-3}$ Watt, and assume that the efficiency of transmitter power is $\eta=0.5$ at the BS. These above parameters are obtained from the results of $[19,37]$ for hybrid architectures.

Figure 5 displays the total achievable EE as a function of the input SNR. These curves are shown for hybrid architectures based on DFT processing, ideal phase shifters, and switch network. It is shown that the total achievable EE of hybrid architectures based on DFT processing is always superior to full digital architectures, as well as hybrid architectures ideal phase shifters and switch network. This is because the DFT processing has low cost and uses a few number of RF chains. However, the total achievable EE of the architectures with ideal phase shifters is better than that of the architectures with switch network for the reason that the degradation of the achievable SE with switch network as compared with the ideal phase shifters case is significant, especially for switch network case. Furthermore, the total achievable EE of hybrid architectures with DFT processing invariably outperforms the other in the input SNR regime, which indicates that the DFT processing is the best one, because the energy consumption of DFT processing is very small. In addition, it is shown that the total achievable EE first improves the input SNR and then reduces while the input SNR increases. Indeed, there exists the best input SNR that makes the total achievable EE of system maximization, which is consistent with analytical results in Corollary 5.

Figure 6 depicts the total achievable EE versus the number of BS antennas and includes full digital architecture and hybrid architectures with DFT processing, ideal phase shifters, and switch network. It is shown that the total achievable EE of hybrid architectures with DFT processing decreases by increasing the number of BS antennas. This is because keeping the number of RF chains and the SNR fixed, the total achievable SE becomes a constant, but the total energy consumption increases by increasing the number of BS antennas. Finally, this leads to the total achievable EE of hybrid architectures with DFT processing decreases. Obviously, the total achievable EE with DFT processing outperforms that of other hybrid architectures. For the hybrid architectures with ideal phase shifters and switch network, it is shown that the best number of BS antennas exists that makes the total achievable EE optimum, which is consistent with the conclusion described in [45]. For full digital architectures, it is shown that as the number of BS antennas increases, the total achievable EE decreases. This is because each antenna needs to deploy a unique RF chain, which is augmented by increasing the number of BS antennas.

\section{Conclusion}

In this paper, low cost and high efficiency hybrid massive MIMO system has been studied, in which the analog domain uses DFT processing and the digital domain utilizes ZF precoding. We derived the exact expressions on the achievable SE. Results showed that compared with full digital architecture and hybrid architecture with ideal phase shifters, there is some degradation of achievable SE for hybrid architecture with DFT processing. Fortunately, the degraded achievable SE can be compensated by providing the more number of RF chains and the input SNR. Moreover, we also studied the total achievable EE and derived exact expression of the achievable EE. Compared to conventional massive MIMO system, regardless of full digital and hybrid architecture, results showcased that the total achievable EE of hybrid architectures with DFT processing invariably outperforms the full digital architectures and hybrid architectures with switch network and ideal phase shifters. We also found out that the achievable EE decreases as the number of antennas chains increases, while the best SNR exists that realizes the total achievable EE optimum. We believe that the hybrid massive MIMO systems with DFT processing have low cost and high efficiency for $5 \mathrm{G}$ communication system. In the future, we will incorporate some other wireless techniques such as [46-49] to further enhance the performance of the massive MIMO system.

\section{Appendix}

\section{A. Proof of Theorem 2}

To calculate the exact expression on the achievable SE in (5), we shall recall that the definition of $\mathbf{G}_{e q}=\mathbf{G}^{H} \mathbf{F}$ with $\mathbf{G}=\mathbf{H D}^{1 / 2}$. By plugging these results into (8), the achievable ergodic SE in (8) is expressed as

$$
R_{k}=\mathbb{E}\left\{\log _{2}\left(1+\frac{P \beta_{k}}{\left[\mathbf{H}^{H} \mathbf{A H}\right]_{k, k}^{-1}}\right)\right\} .
$$

For convenience, we firstly define an auxiliary matrix A, which is identical to $\mathbf{A}=\mathbf{F} \mathbf{F}^{H}$. According to the properties of the DFT matrix, we easily know that the column vectors of the DFT matrix are mutually orthogonal, and then the auxiliary matrix $\mathbf{A}$ is transformed by the eigen-decomposition, which can be written as

$$
\mathbf{A}=\mathbf{F} \mathbf{F}^{H}=\mathbf{U} \boldsymbol{\Lambda} \mathbf{U}^{H},
$$

where $\Lambda$ is the diagonal matrix, whose entries are one or zero. Since the partial matrix $\mathbf{F}$ is a $N_{t} \times N_{s}$ dimension of 
DFT, by applying the eigen-decomposition $\Lambda$ is a diagonal matrix having $N_{s}$ unit singular values, whose diagonal entries can be expressed as $\boldsymbol{\Lambda}=\operatorname{diag}\{1, \cdots, 1,0, \cdots, 0\}$. By applying the unitary transformation, the matrix $\mathbf{H}^{H} \mathbf{A H}$ has the same distribution form as $\mathbf{T}^{H} \mathbf{T}$, where $\mathbf{T}$ reduces to a complex Gaussian matrix of $M \times N_{s}$ dimension and is equivalent to $N_{s}$ columns of $\mathbf{H}$. Thus, (A.1) can be written as

$$
R_{k}=\mathbb{E}\left\{\log _{2}\left(1+\frac{P \beta_{k}}{\left[\mathbf{T}^{H} \mathbf{T}\right]_{k, k}^{-1}}\right)\right\} .
$$

We define

$$
X_{k}=\frac{1}{\left[\left(\mathbf{T}^{H} \mathbf{T}\right)\right]_{k, k}^{-1}}
$$

Thus, (A.3) can be rewritten as

$$
R_{k}=\mathbb{E}\left\{\log _{2}\left(1+P \beta_{k} X_{k}\right)\right\} .
$$

We study the evaluation of $R_{k}$ according to the following expression:

$$
R_{k}=\log _{2}(e) \int_{0}^{\infty} \ln \left(1+P \beta_{k} x_{k}\right) p\left(x_{k}\right) d x_{k} .
$$

According to random matrix theory, when the entries of small-scale fading $\mathbf{T}$ are i.i.d. Rayleigh random variances [5052], the p.d.f. of $X_{k}$ is given by

$$
p\left(x_{k}\right)=\frac{e^{-x_{k}}}{\left(N_{s}-M\right) !} x_{k}^{N_{s}-M} .
$$

Substituting (A.7) into (A.6) and employing the following integration identity:

$$
\begin{aligned}
& \int_{0}^{\infty} \ln (1+a \lambda) \lambda^{q-1} e^{-b \lambda} d y \\
& \quad=(q-1) ! e^{b / a} b^{-q} \sum_{h=1}^{q} E_{h}\left(\frac{b}{a}\right) .
\end{aligned}
$$

By doing more basic mathematical manipulations, we finish the detailed proof.

\section{B. Proof of Corollary 4}

For fairness among users, we assume that the large-scale fading of each user is identical, namely, $\beta_{k}=\beta, \forall M$. According to the achievable SE of per user (9), the total achievable SE of system can be expressed as

$$
\bar{R}_{\text {sum }}=M \log _{2}\left(1+P \beta\left(N_{s}-M+1\right)\right) .
$$

We differentiate the total achievable SE in (B.1) in terms of random variable $M$, the first-order partial derivative of $\bar{R}_{\text {sum }}(M)$ is calculated as

$$
\begin{aligned}
& \frac{\bar{R}_{\text {sum }}(M)}{\partial M} \\
& =\frac{\left(\ln \left(1+P \beta\left(N_{s}-M+1\right)\right)-P \beta M /\left(1+P \beta\left(N_{s}-M+1\right)\right)\right)}{\ln 2} .
\end{aligned}
$$

Since the second term is negative, it is hard to judge directly if the value of (B.2) is negative or positive, and we need to further solve the second order partial derivative of $\bar{R}_{\text {sum }}(M)$. The detailed computation and associated analysis of secondorder partial can be found in the works [53-55]. The secondorder partial derivative of $\bar{R}_{\text {sum }}(M)$ is calculated as

$$
\begin{gathered}
\frac{\bar{R}_{\text {sum }}^{2}(M)}{\partial M^{2}}=\left(\frac{-P \beta M}{1+P \beta\left(N_{s}-M+1\right)}\right. \\
\left.+\frac{-P \beta\left(N_{s}+P+1\right)}{\left(1+P \beta\left(N_{s}-M+1\right)\right)^{2}}\right) .
\end{gathered}
$$

We found that $\bar{R}_{\text {sum }}^{2}(M) / \partial M^{2}<0$ due to the value of numerator is a negative; this shows that the function $\bar{R}_{\text {sum }}(M)$ is concave with respect to $M$. According to convex optimization theory, it is easy to know that a unique globally optimal number of users always exist, which enables us to achieve the total achievable SE maximization. To check the optimal value, let the first-order partial derivative be equal to zero that holds

$$
M^{o p t}=\left\{M \mid \frac{\partial \widetilde{R}_{\text {sum }}(M)}{\partial M}=0\right\} .
$$

Plugging (B.2) into (B.4), we attain

$$
\ln \left(1+P \beta\left(N_{s}-M+1\right)\right)=\frac{P \beta}{1+P \beta\left(N_{s}-M+1\right)} .
$$

In order to solve the above equation in terms of random variable $M$, we observe that the equation is so sophisticated because it involves the logarithmic function. We start by letting $y=1+P \beta\left(N_{s}-M+1\right)$; (B.5) can be simplified as

$$
\begin{aligned}
(1 & \left.+P \beta\left(N_{s}+1\right)\right) \exp (1) \\
& =\frac{1+P \beta\left(N_{s}+1\right)}{y} \cdot \exp \left(\frac{1+P \beta\left(N_{s}+1\right)}{y}\right) .
\end{aligned}
$$

By applying the properties of the Lambert function [41] that holds $x=W_{0}(x) \exp \left(W_{0}(x)\right)$, we can attain the following equation:

$$
y=\frac{1+P \beta\left(N_{s}+1\right)}{W_{0}\left(1+P \beta\left(N_{s}+1\right) \exp (1)\right)}
$$

Substituting $y$ into (B.7) and playing some basic mathematical transformations, we finish the proof.

\section{Data Availability}

The authors derived the writing material from different journals as provided in the references. A MATLAB tool has been utilized to simulate the concept.

\section{Conflicts of Interest}

The authors declare that there are no conflicts of interest regarding the publication of this paper. 


\section{Acknowledgments}

This work was supported in part by the Natural Science Foundation of Guangdong Province of China under Grant 2018A030310338, the National Natural Science Foundation of China under Grants 61671144, 61801132, and 61871139, the Project of Educational Commission of Guangdong Province of China under Grant 2017KQNCX155, the Guangdong Natural Science Funds for Distinguished Young Scholar under Grant 2014A030306027, the Innovation Team Project of Guangdong Province University under Grant 2016KCXTD017, the Science and Technology Program of Guangzhou under Grant 201807010103, the Project of Fundamental Science and Frontier Technology Research Project of Chongqing under Grant cstc2017jcyjAX0380, the Guangdong Science and Technology Project under Grant 2017A050506058, the Major Research Project of Guangdong Provincial Department of Education under Grant 2016KZDXM035, the Innovation Group Project of Guangzhou under Grant 1201610010, and the Yangcheng Scholars Project of Guangzhou under Grant 1201561560.

\section{References}

[1] F. Zhou, L. Fan, X. Lei, G. Luo, H. Zhang, and J. Zhao, "Edge Caching With Transmission Schedule for Multiuser Multirelay Networks," IEEE Communications Letters, vol. 22, no. 4, pp. 776-779, 2018.

[2] W. Tan, W. Huang, X. Yang, W. Liu, and L. Fan, "Multiuser precoding scheme and achievable rate analysis for massive," EURASIP Journal on Wireless Communications and Networking, 2018.

[3] B. Liu, Q. Zhu, W. Tan, and H. Zhu, "Congestion-Optimal WiFi Offloading with User Mobility Management in Smart Communications," Wireless Communications and Mobile Computing, vol. 2018, pp. 1-15, 2018.

[4] H. Huang, J. Xia, X. Liu et al., "Switch-and-stay combining for energy harvesting relaying systems," Physical Communication, vol. 28, pp. 28-34, 2018.

[5] F. Rusek, D. Persson, B. K. Lau et al., "Scaling up MIMO: opportunities and challenges with very large arrays," IEEE Signal Processing Magazine, vol. 30, no. 1, pp. 40-60, 2013.

[6] F. Zhou, M. Du, Y. Wang, and G. Luo, "Joint source-channel coding for band-limited backhauls in coordinated multi-point systems," IET Communications, vol. 10, no. 13, pp. 1562-1570, 2016.

[7] R. Zhao, Y. Yuan, L. Fan, and Y.-C. He, "Secrecy Performance Analysis of Cognitive Decode-and-Forward Relay Networks in Nakagami-m Fading Channels," IEEE Transactions on Communications, vol. 65, no. 2, pp. 549-563, 2017.

[8] J. Yuan, S. Jin, W. Xu, W. Tan, M. Matthaiou, and K.-K. Wong, "User-Centric Networking for Dense C-RANs: HighSNR Capacity Analysis and Antenna Selection," IEEE Transactions on Communications, vol. 65, no. 11, pp. 5067-5080, 2017.

[9] L. Lu, G. Y. Li, A. L. Swindlehurst, A. Ashikhmin, and R. Zhang, "An overview of massive MIMO: benefits and challenges," IEEE Journal of Selected Topics in Signal Processing, vol. 14, no. 5, pp. 136-146, 2014.

[10] J. Xia, F. Zhou, X. Lai et al., "Cache Aided Decode-andForward Relaying Networks: From the Spatial View," Wireless
Communications and Mobile Computing, vol. 2018, Article ID 5963584, 9 pages, 2018.

[11] X. Lai, J. Xia, M. Tang, H. Zhang, and J. Zhao, "Cache-aided multiuser cognitive relay networks with outdated channel state information," IEEE Access, vol. 6, pp. 21879-21887, 2018.

[12] W. Tan, S. Jin, C. Wen, and T. Jiang, "Spectral efficiency of multi-user millimeter wave systems under single path with uniform rectangular arrays," EURASIP Journal on Wireless Communications and Networking, vol. 181, pp. 1-13, 2017.

[13] H. Shuangfeng, I. Chih-Lin, X. Zhikun, and C. Rowell, "Largescale antenna systems with hybrid analog and digital beamforming for millimeter wave 5G," IEEE Communications Magazine, vol. 53, no. 1, pp. 186-194, 2015.

[14] O. E. Ayach, S. Rajagopal, S. Abu-Surra, Z. Pi, and R. W. Heath, "Spatially sparse precoding in millimeter wave MIMO systems," IEEE Transactions on Wireless Communications, vol. 13, no. 3, pp. 1499-1513, 2014.

[15] A. Alkhateeb, O. El Ayach, G. Leus, and R. W. Heath, "Channel estimation and hybrid precoding for millimeter wave cellular systems," IEEE Journal of Selected Topics in Signal Processing, vol. 8, no. 5, pp. 831-846, 2014.

[16] Chanhong K., Taeyoung K., and Ji-Yun S., "Multi-beam transmission diversity with hybrid beamforming for MIMO-OFDM systems," in Proceedings of the 2013 IEEE Globecom Workshops (GC Wkshps), pp. 61-65, Atlanta, GA, December 2013.

[17] Z. Song, Z. Zhang, X. Liu, Y. Liu, and L. Fan, "Simultaneous cooperative spectrum sensing and wireless power transfer in multi-antenna cognitive radio," Physical Communication, vol. 29, pp. 78-85, 2018.

[18] R. Zi, X. Ge, J. Thompson, C.-X. Wang, H. Wang, and T. Han, "Energy Efficiency Optimization of 5G Radio Frequency Chain Systems," IEEE Journal on Selected Areas in Communications, vol. 34, no. 4, pp. 758-771, 2016.

[19] R. Mendez-Rial, C. Rusu, N. Gonzalez-Prelcic, A. Alkhateeb, and R. W. Heath, "Hybrid MIMO Architectures for Millimeter Wave Communications: Phase Shifters or Switches?" IEEE Access, vol. 4, pp. 247-267, 2016.

[20] A. Garcia-Rodriguez, V. Venkateswaran, P. Rulikowski, and C. Masouros, "Hybrid Analog-Digital Precoding Revisited Under Realistic RF Modeling," IEEE Wireless Communications Letters, vol. 5, no. 5, pp. 528-531, 2016.

[21] J. Butler and R. Lowe, "Beam forming matrix simplifies design of electronically scanned antennas," Electron. Design, pp. 170$173,1961$.

[22] H. A. Darwish and I. Hartimo, "Aclose accord on DFT based frequency and phasor estimators for numerical relays," Materials Research Bulletin, pp. 29-36, 2001.

[23] H. A. Darwish and M. Fikri, "Practical considerations for recursive DFT implementation in numerical relays," IEEE Transactions on Power Delivery, vol. 22, no. 1, pp. 42-49, 2007.

[24] W. Tan, M. Matthaiou, S. Jin, and X. Li, "Spectral Efficiency of DFT-Based Processing Hybrid Architectures in Massive MIMO," IEEE Wireless Communications Letters, vol. 6, no. 5, pp. 586-589, 2017.

[25] D. Deng, C. Li, L. Fan, X. Liu, and F. Zhou, "Impact of Antenna Selection on Physical-Layer Security of NOMA Networks," Wireless Communications and Mobile Computing, vol. 2018, Article ID 2390834, 11 pages, 2018.

[26] F. Shi, L. Fan, X. Liu, Z. Na, and Y. Liu, "Probabilistic Caching Placement in the Presence of Multiple Eavesdroppers," Wireless Communications and Mobile Computing, vol. 2018, Article ID 2104162, 10 pages, 2018. 
[27] D. Deng, M. Yu, J. Xia, Z. Na, J. Zhao, and Q. Yang, "Wireless powered cooperative communications with direct links over correlated channels," Physical Communication, vol. 28, pp. 147153, 2018.

[28] L. Liang, W. Xu, and X. Dong, "Low-complexity hybrid precoding in massive multiuser MIMO systems," IEEE Wireless Communications Letters, vol. 3, no. 6, pp. 653-656, 2014.

[29] S. Suh, A. Basu, C. Schlottmann, P. E. Hasler, and J. R. Barry, "Low-power discrete Fourier transform for OFDM: a programmable analog approach," IEEE Transactions on Circuits and Systems I: Regular Papers, vol. 58, no. 2, pp. 290-298, 2011.

[30] A. F. Molisch, X. Zhang, S. Y. Kung, and J. Zhang, "DFTbased hybrid antenna selection schemes for spatially correlated MIMO channels," in Proceedings of the 14th IEEE 2003 International Symposium on Personal, Indoor and Mobile Radio Communications, PIMRC2003, pp. 1119-1123, China, September 2003.

[31] H. Yang and T. L. Marzetta, "Performance of conjugate and zeroforcing beamforming in large-scale antenna systems," IEEE Journal on Selected Areas in Communications, vol. 31, no. 2, pp. 172-179, 2013.

[32] Y.-G. Lim, C.-B. Chae, and G. Caire, "Performance Analysis of Massive MIMO for Cell-Boundary Users," IEEE Transactions on Wireless Communications, vol. 14, no. 12, pp. 6827-6842, 2015.

[33] M. Vu and A. Paulraj, "MIMO wireless linear precoding," IEEE Signal Processing Magazine, vol. 24, no. 5, pp. 86-105, 2007.

[34] M. Abramowitz and I. A. Stegun, Handbook of Mathematical Functions, New York: Dover, 1974.

[35] C. Li, Y. Li, K. Song, and L. Yang, "Energy efficient design for multiuser downlink energy and uplink information transfer in 5G," Science China Information Sciences, vol. 59, no. 2, pp. 1-8, 2016.

[36] C. Li, K. Song, D. Wang, F.-C. Zheng, and L. Yang, "Optimal remote radio head selection for cloud radio access networks," Science China Information Sciences, vol. 59, no. 10, pp. 59-73, 2016.

[37] E. Björnson, L. Sanguinetti, J. Hoydis, and M. Debbah, “Optimal design of energy-efficient multi-user MIMO systems: Is massive MIMO the answer?" IEEE Transactions on Wireless Communications, vol. 14, no. 6, pp. 3059-3075, 2015.

[38] J. Li, M. Wen, X. Jiang, and W. Duan, "Space-Time MultipleMode Orthogonal Frequency Division Multiplexing with Index Modulation," IEEE Access, vol. 5, pp. 23212-23222, 2017.

[39] C. Kong, C. Zhong, M. Matthaiou, and Z. Zhang, "Performance of downlink massive MIMO in ricean fading channels with ZF precoder," in Proceedings of the IEEE International Conference on Communications, ICC 2015, pp. 1776-1782, UK, June 2015.

[40] E. Björnson, L. Sanguinetti, J. Hoydis, and M. Debbah, "Designing multi-user MIMO for energy efficiency: When is massive MIMO the answer?" in Proc. IEEE WCNC, pp. 56-64, 2014.

[41] R. M. Corless, G. H. Gonnet, D. E. G. Hare, and D. E. Knuth, "On the lambert W function, Advances in Computational Mathem," Advances in Computational Mathematics, vol. 5, no. 1, pp. 329359, 1996.

[42] Y. Lv, H. Wu, Y. Liu et al., "Quantitative research on the influence of particle size and filling thickness on aerogel glazing performance," Energy and Buildings, vol. 174, pp. 190-198, 2018.

[43] H. Q. Ngo, E. G. Larsson, and T. L. Marzetta, "Energy and spectral efficiency of very large multiuser MIMO systems," IEEE Transactions on Communications, vol. 61, no. 4, pp. 1436-1449, 2013.
[44] X. Gao, L. Dai, S. Han, I. Chih-Lin, and R. W. Heath, "Energyefficient hybrid analog and digital precoding for MmWave MIMO systems with large antenna arrays," IEEE Journal on Selected Areas in Communications, vol. 34, no. 4, pp. 998-1009, 2016.

[45] C. Sun, X. Gao, and Z. Ding, "Spectral efficiency and power allocation for mixed-ADC massive MIMO system," IEEE Transactions on Signal Processing, vol. 65, no. 11, pp. 2962-2974, 2017.

[46] W. Zhou, D. Deng, J. Xia, and Z. Shao, “The Precoder Design with Covariance Feedback for Simultaneous Information and Energy Transmission Systems," Wireless Communications and Mobile Computing, vol. 2018, Article ID 8472186, 17 pages, 2018.

[47] D. Deng, X. Li, L. Fan, W. Zhou, R. Qingyang Hu, and Z. Zhou, "Secrecy Analysis of Multiuser Untrusted Amplify-andForward Relay Networks," Wireless Communications and Mobile Computing, vol. 2017, Article ID 9580639, 11 pages, 2017.

[48] X. Liu, X. Zhang, M. Jia, L. Fan, W. Lu, and X. Zhai, "5Gbased green broadband communication system design with simultaneous wireless information and power transfer," Physical Communication, vol. 28, pp. 130-137, 2018.

[49] L. Fan, R. Zhao, F.-K. Gong, N. Yang, and G. K. Karagiannidis, "Secure Multiple Amplify-and-Forward Relaying over Correlated Fading Channels," IEEE Transactions on Communications, vol. 65, no. 7, pp. 2811-2820, 2017.

[50] X. Wang, H. Zhang, L. Fan, and Y. Li, "Performance of Distributed Switch-and-Stay Combining for Cognitive Relay Networks with Primary Transceiver," Wireless Personal Communications, vol. 97, no. 2, pp. 3031-3042, 2017.

[51] Z. Na, Y. Wang, X. Li et al., "Subcarrier allocation based Simultaneous Wireless Information and Power Transfer algorithm in 5G cooperative OFDM communication systems," Physical Communication, vol. 29, pp. 164-170, 2018.

[52] M. Zhao, D. Deng, W. Zhou, and L. Fan, "Non-renewable energy efficiency optimization in energy harvesting relayassisted system," Physical Communication, vol. 29, pp. 183-190, 2018.

[53] Y. Liang, H. Wu, G. Huang, J. Yang, and H. Wang, “Thermal performance and service life of vacuum insulation panels with aerogel composite cores," Energy and Buildings, vol. 154, pp. 606-617, 2017.

[54] Y. Jian, H. Wu, G. Huang, Y. Liang, and Y. Liao, "Modeling and coupling effect evaluation of thermal conductivity of ternary opacifier/fiber/aerogel composites for super-thermal insulation," Materials Design, vol. 133, no. 5, pp. 224-236, 2017.

[55] J. Yang, H. Wu, M. Wang, and Y. Liang, "Prediction and optimization of radiative thermal properties of nano $\mathrm{TiO} 2$ assembled fibrous insulations," International Journal of Heat and Mass Transfer, vol. 117, pp. 729-739, 2018. 


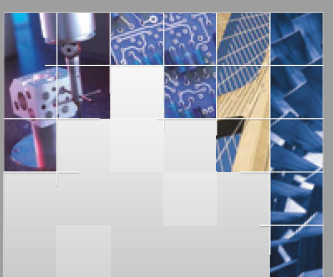

\section{Enfincering}
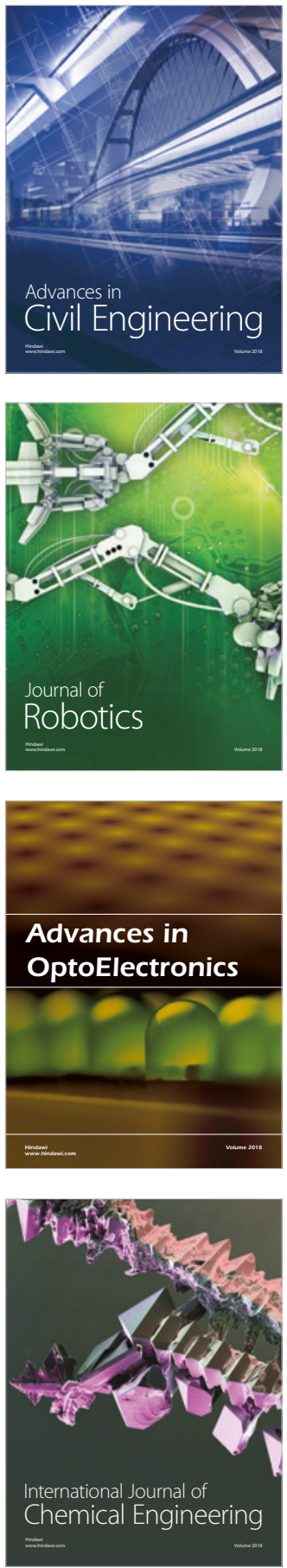

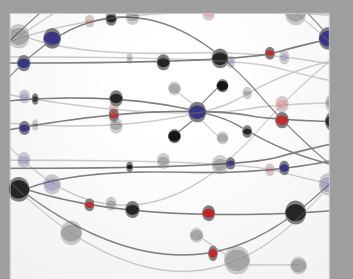

\section{Rotating \\ Machinery}

The Scientific World Journal

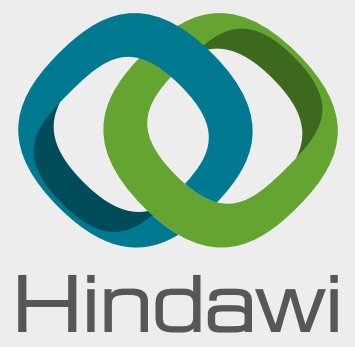

Submit your manuscripts at

www.hindawi.com
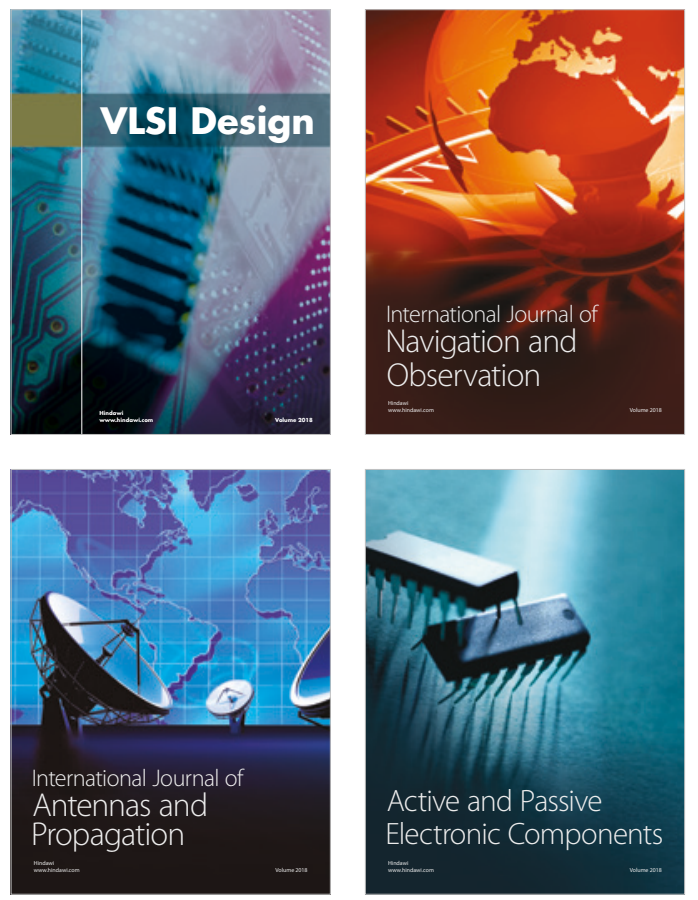
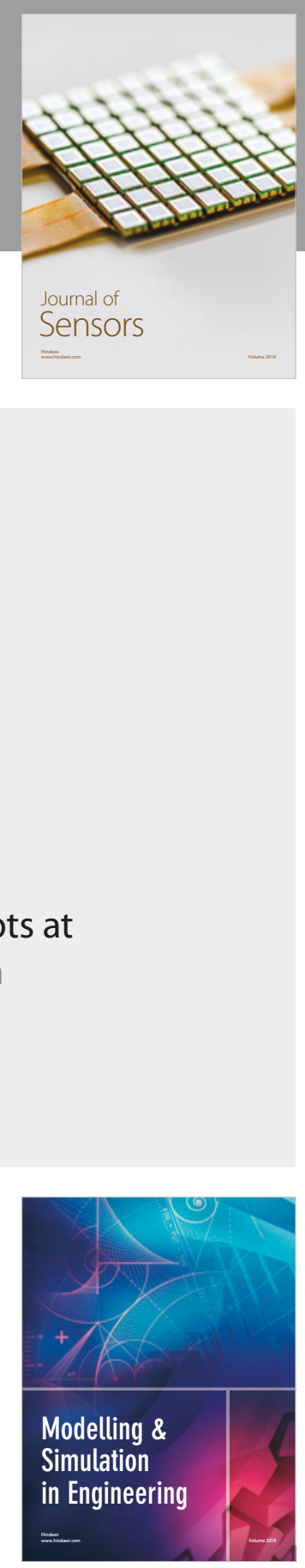

\section{Advances \\ Multimedia}
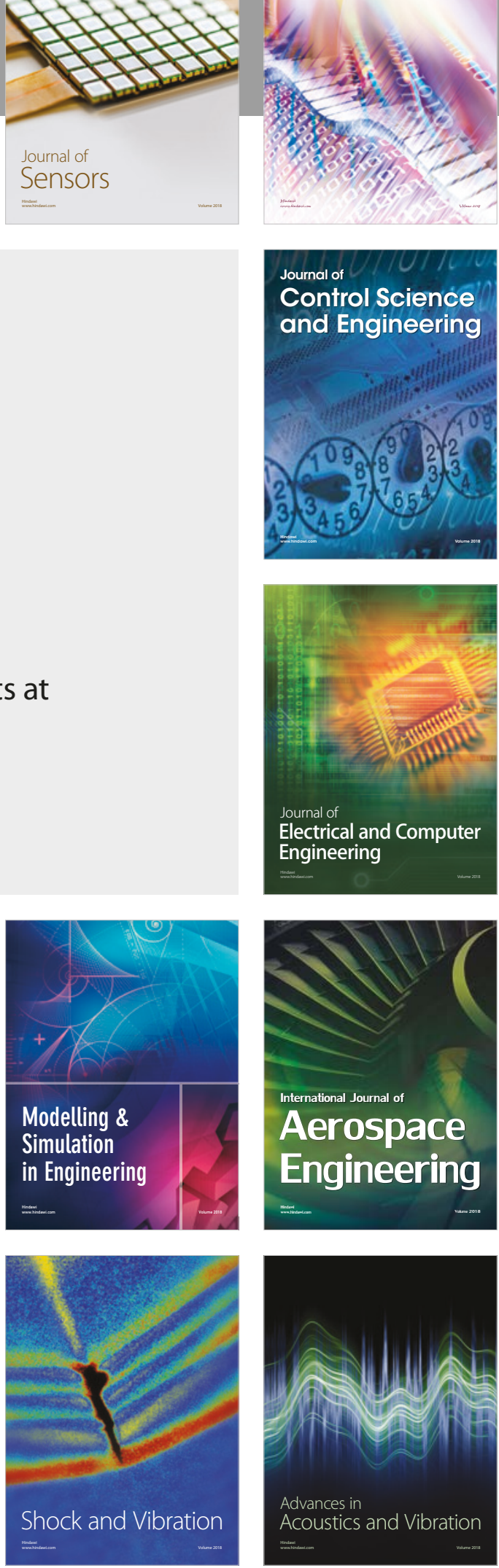